\title{
Elucidating Electron Storage and Distribution within the Pentaheme Scaffold of Cytochrome $c$ Nitrite Reductase (NrfA)
}

Victor Sosa Alfaro, ${ }^{1}$ Julius Campeciño, ${ }^{2}$ Matthew Tracy, $^{3}$ Sean J. Elliott, ${ }^{3 *}$ Eric L. Hegg, ${ }^{2 *}$ Nicolai Lehnert, ${ }^{1 *}$

${ }^{1}$ Department of Chemistry, The University of Michigan, Ann Arbor, Michigan 48109, United States. Email: lehnertn@umich.edu

${ }^{2}$ Department of Biochemistry \& Molecular Biology, Michigan State University, East Lansing, Michigan 48824, United States. Email: EricHegg@msu.edu

${ }^{3}$ Department of Chemistry, Boston University, Boston, Massachusetts 02215, United States. Email: elliott@bu.edu

\section{Supporting Information}


Residuals for fits of the oxidative and reductive scans of WT NrfA and H108M NrfA 
NrfA EPR titration respective difference spectra

$\begin{array}{lr}\text { Power dependence of NrfA EPR signal } & \text { S20 }\end{array}$

$\begin{array}{ll}\text { Power dependence plot of NrfA EPR signal } & \text { S21 }\end{array}$

Overlay of NrfA taken at microwave power $5 \mathrm{~mW}$ with simulation

Overlay of the normalized EPR spectra from Figure S19

Calculated $n$ values for WT NrfA and H108M NrfA

S24

Midpoint potential values for WT NrfA and H108M NrfA 


\begin{abstract}
ATGAAATACCTGCTGCCGACCGCTGCTGCTGGTCTGCTGCTCCTCGCTGCCCAGCCGGCGATGGCCGCGC CGCCGAAAGCGGAACAAGCGAAGATTGCGGAGATCCCGGATGGCACCATTGACCCGGCGGTTTGGGGC AAGAACTACCCGGAGGAGTACCAGACCTGGAAAGACACCGCGCTGCCGACCCCGGAAGGCAAGAGCAA ATATAAGAAAGGCAACGACGGTGGCAAGGTTTACGATAAACTGAGCGAGTATCCGTTCATCGCGCTGCT GTTTAACGGCTGGGGTTTCGGCATTGAGTACAACGAACCGCGTGGTCACGTGTATATGATGAAGGACCA GAAAGAAATTGATCCGAGCCGTCTGAAGGGTGGCGGTGCGTGCCTGACCTGCAAGACCCCGTACGCGCC GCAGCTGGCGCAGAAGCAAGGTGTTACCTACTTTAGCCAAAGCTATGCGGATGCGGTGAACCAGATCCC GAAAGAGCACCAAGAAATGGGCGTTGCGTGCATTGACTGCCACAACAACAAGGATATGGGTCTGAAAAT CAGCCGTGGCTTCACCCTGGTTAAGGCGCTGGACAAAATGGGTGTGGATCAGACCAAGCTGACCAACCA AGACAAACGTAGCCTGGTTTGCGCGCAGTGCCACGTGACCTACACCATTCCGAAGGATGCGAACATGAA AAGCCAAGACGTTTTCTTTCCGTGGGATGAGAGCAAGTGGGGCAAAATCAGCATCGAAAACATCATTAA GAAAATGCGTAGCGACAAGAGCTATGGTGAATGGACCCAGGCGGTGACCGGCTTTAAAATGGCGTACA TCCGTCACCCGGAGTTCGAAATGTATAGCAACCAAAGCGTTCACTGGATGGCGGGTGTGAGCTGCGCGG ATTGCCACATGCCGTACACCAAAGTGGGCAGCAAGAAAATCAGCGACCACCGTATTATGAGCCCGCTGA AAAACGATTTTAAGGGTTGCAAACAGTGCCACAGCGAGAGCAGCGAATGGCTGAAGAACCAGGTTATCA CCATTCAAGACCGTGCGGCGAGCCAATACATTCGTAGCGGTTATGCGCTGGCGACCGTGGCGAAGCTGT TCGAGATGACCCACAAACAGCAAGCGGCGGGCAAGCAGATCGACCAAAAAATGTACGATCAGGCGAAA TTTTACTATGAGGAAGGCTTCTATCGTAACCTGTTCTTTGGTGCGGAGAACAGCATCGGCTTTCACAACCC GACCGAAGCGATGCGTATTCTGGGTGACGCGACCATGTACGCGGGCAAGGCGGATGGCCTGCTGCGTC AAGCGCTGACCAAAGCGGGTGTGGACGTTCCGGTGAAGATCGATCTGGAACTGAGCAAATATACCAACA ACCGTGGCGCGAAAAAGCTGATGTTTAAGCCGGAGCAGGAGCTGAAAGACCCGTATGGCCCGCAGAAG TGGAGCCACCCGCAGTTCGAAAAGTAA
\end{abstract}

Figure S1. The optimized G. lovleyi wild-type NrfA gene sequence (codons in black) fused with the pelB (codons in red) and strep-tag II (codons in blue). 


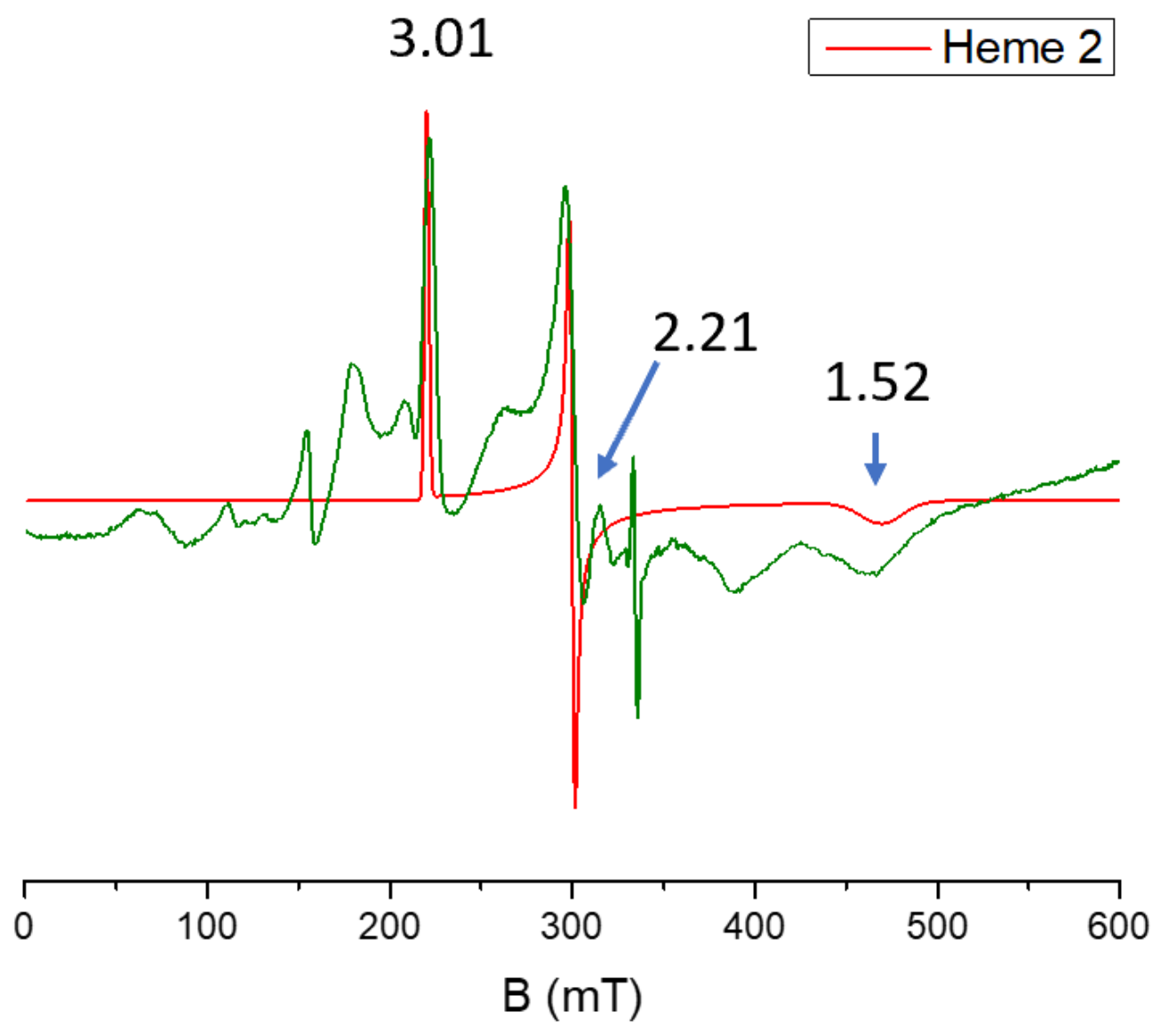

Figure S2. EPR data of as-isolated, fully oxidized and highly purified NrfA (green line, see also Figure 4A), overlaid with a simulation of [Heme 2] (red line). 
(A)

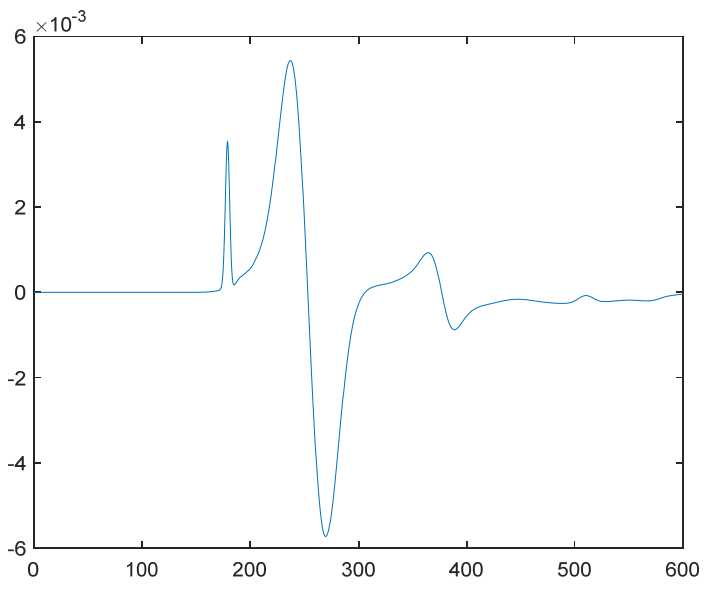

(C)

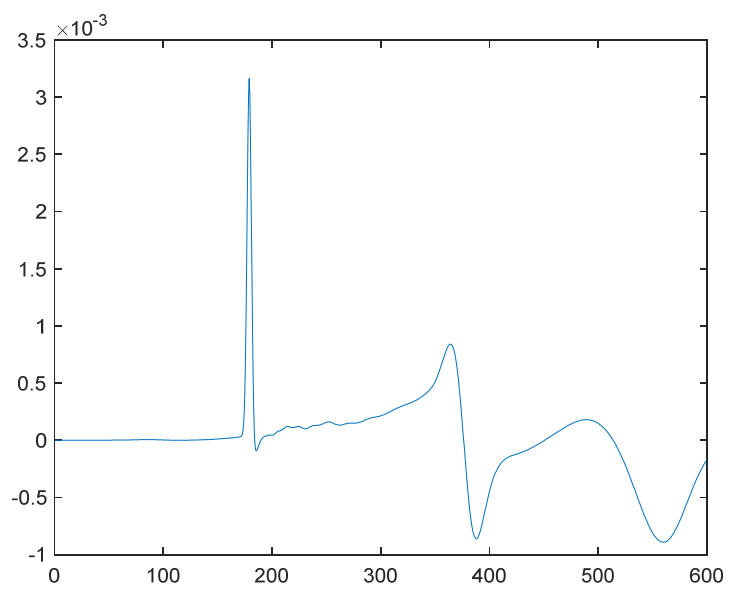

(B)

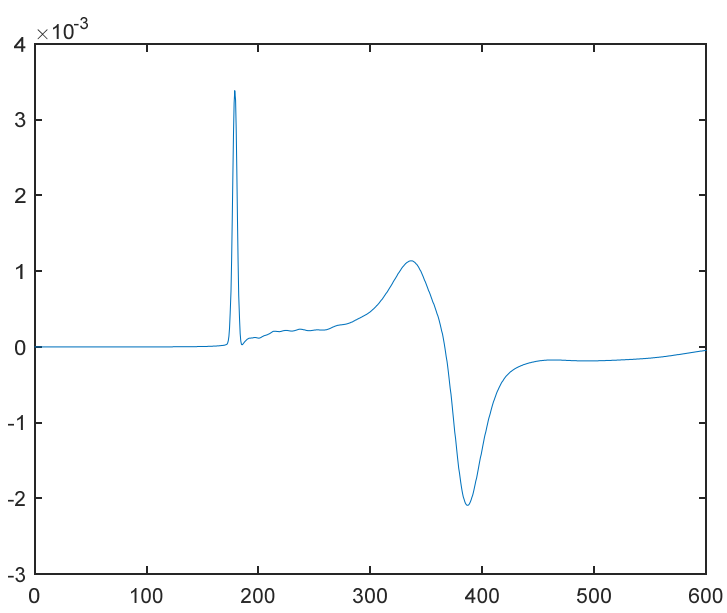

(D)

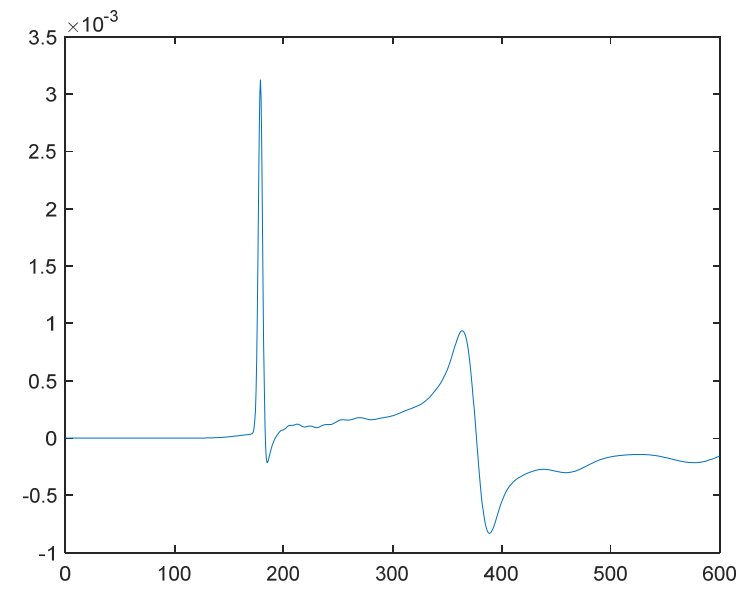

Figure S3. EPR simulations of the Heme 1-3-4 triad. Variation of the $J_{13}$ value (coupling of Hemes 1 and 3) with (A) $J_{13}=0.5 \mathrm{~cm}^{-1}$, (B) $J_{13}=1.0 \mathrm{~cm}^{-1}$, (C) $J_{13}=2.0 \mathrm{~cm}^{-1}$, (D) $J_{13}=3.0 \mathrm{~cm}^{-1}$. All simulations were conducted with: $D=2.5 \mathrm{~cm}^{-1}, E=0.005 \mathrm{~cm}^{-1}, E / D=0.002$, and $J_{34}=0.001 \mathrm{~cm}^{-1}$. For the $g$ values used in the simulations see Table 2. As evident from panels $A-D$, the position and intensity of the derivative-shaped signal at $\sim 250 \mathrm{mT}$ in panel $\mathrm{A}$ is highly dependent on the magnitude of $J_{13}$, allowing us to fit the value of this exchange coupling constant. 
(A)

(B)

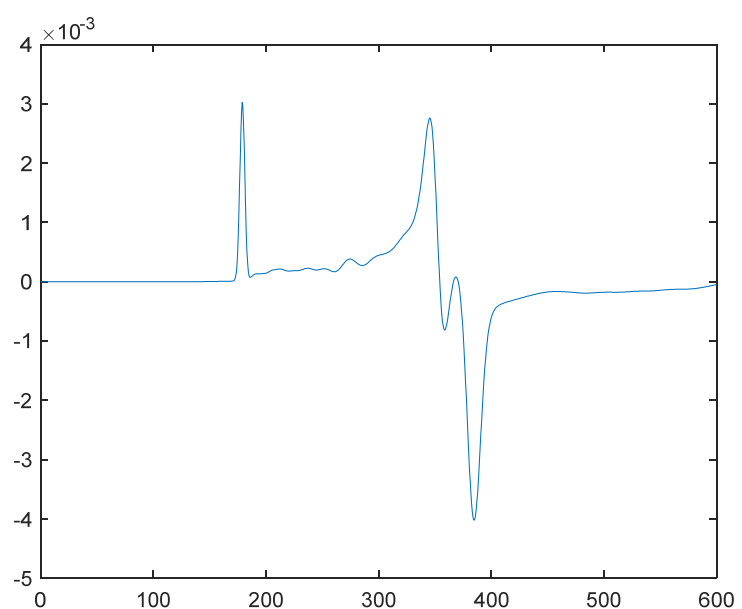

(C)j

(D)

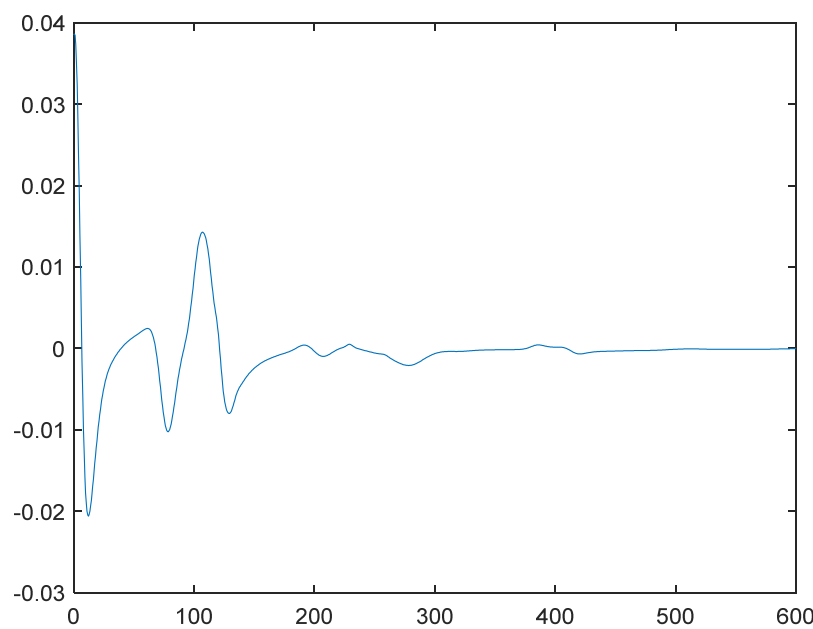

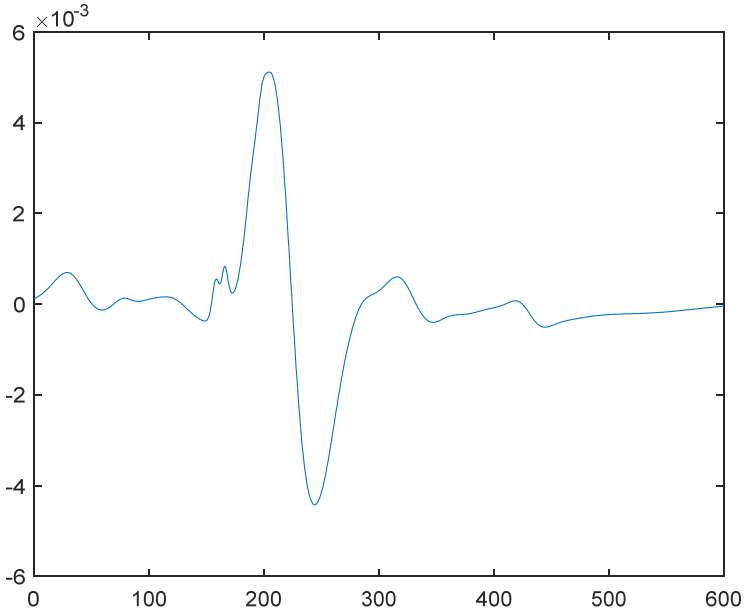

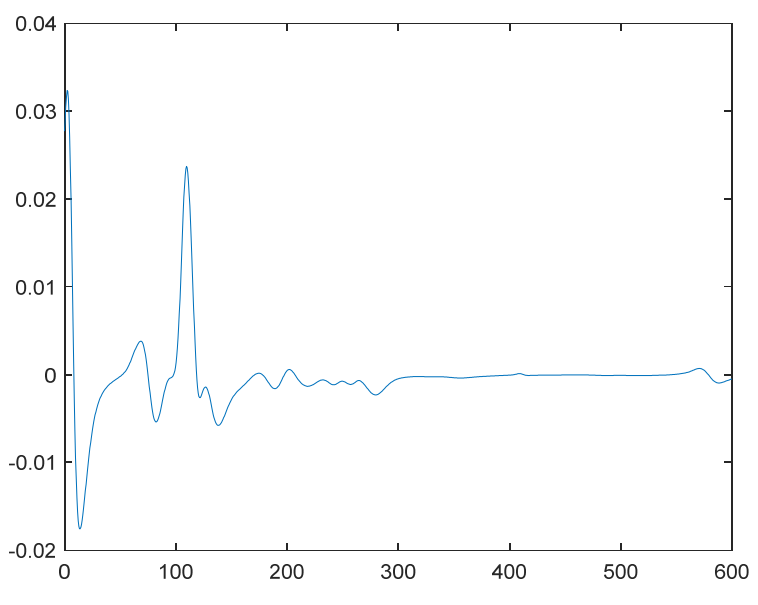

Figure S4. EPR simulations of the Heme 1-3-4 triad. Variation of the $J_{34}$ value (coupling of Hemes 3 and 4) with (A) $J_{34}=0.01 \mathrm{~cm}^{-1}$, (B) $J_{34}=0.1 \mathrm{~cm}^{-1}$, (C) $J_{34}=1.0 \mathrm{~cm}^{-1}$, (D) $J_{34}=2.0 \mathrm{~cm}^{-1}$. All simulations were conducted with: $D=2.5 \mathrm{~cm}^{-1}, E=0.005 \mathrm{~cm}^{-1}, E / D=0.002$, and $J_{13}=0.334 \mathrm{~cm}^{-1}$. For the $g$ values used in the simulations see Table 2. As evident from panels $A-D$, the shape of the spectra is highly dependent on $J_{34}$, allowing us to fit the value of this exchange coupling constant. 
(A)

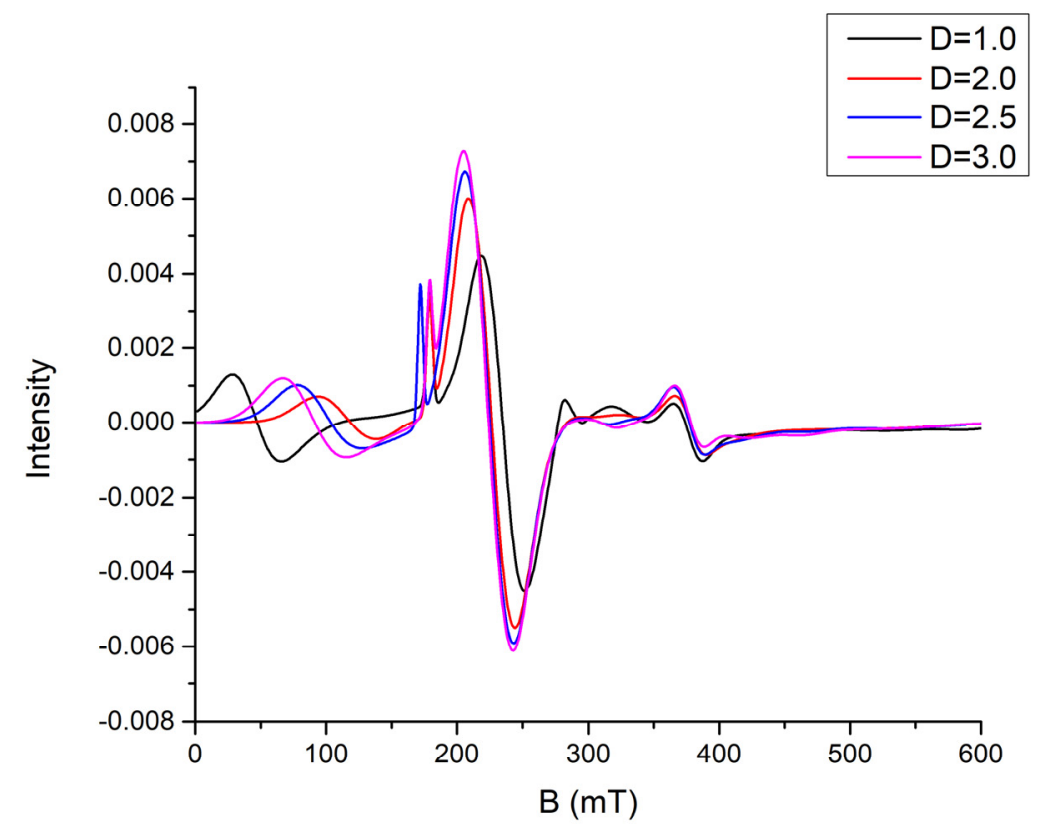

(B)

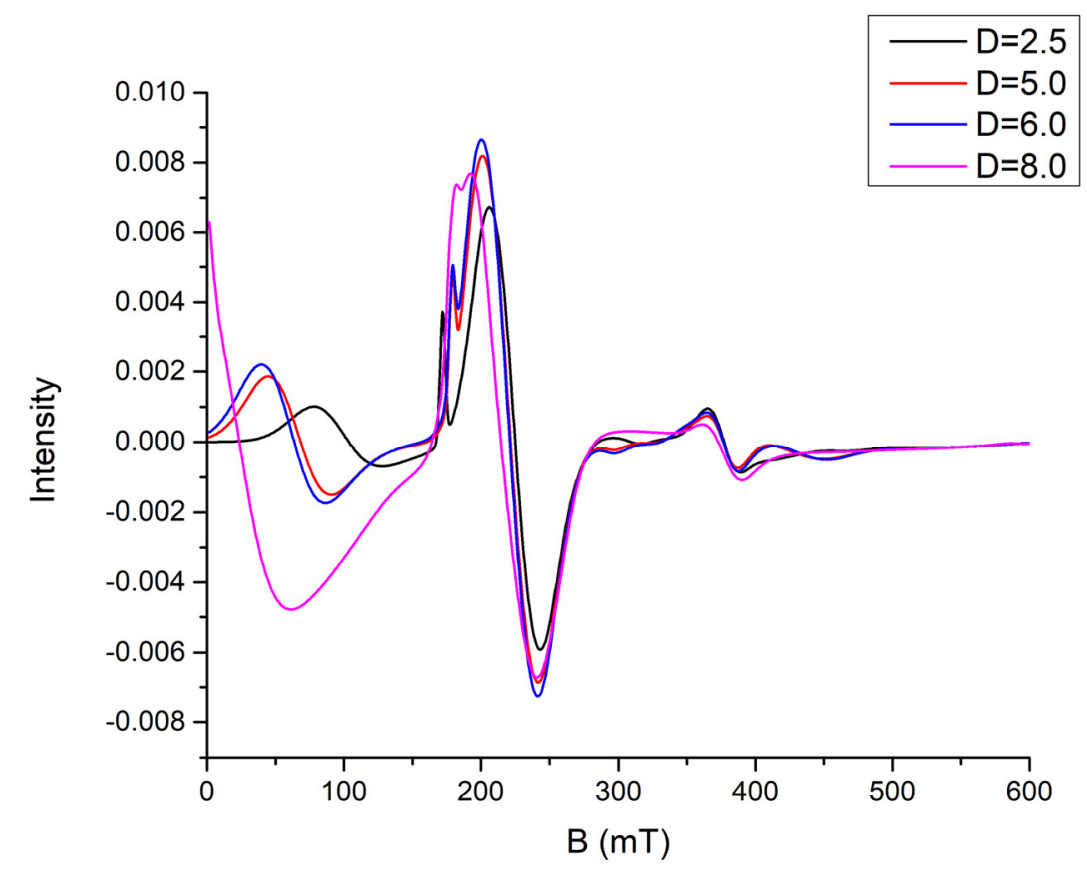

Figure S5. EPR simulations of the Heme 1-3-4 triad. Variation of the $D$ value for Heme $1(\mathrm{~S}=5 / 2)$ between $1-3 \mathrm{~cm}^{-1}$ (A) and $2.5-8 \mathrm{~cm}^{-1}$ (B). All simulations were conducted with: $E=0.005 \mathrm{~cm}^{-1}, J_{13}=$ $0.334 \mathrm{~cm}^{-1}$ and $J_{34}=0.001 \mathrm{~cm}^{-1}$. For the $g$ values used in the simulations see Table 2. As evident from panels $A$ and $B$, the low-field signals are very sensitive to the value of $D$, allowing us to fit the value of this parameter. 
(A)

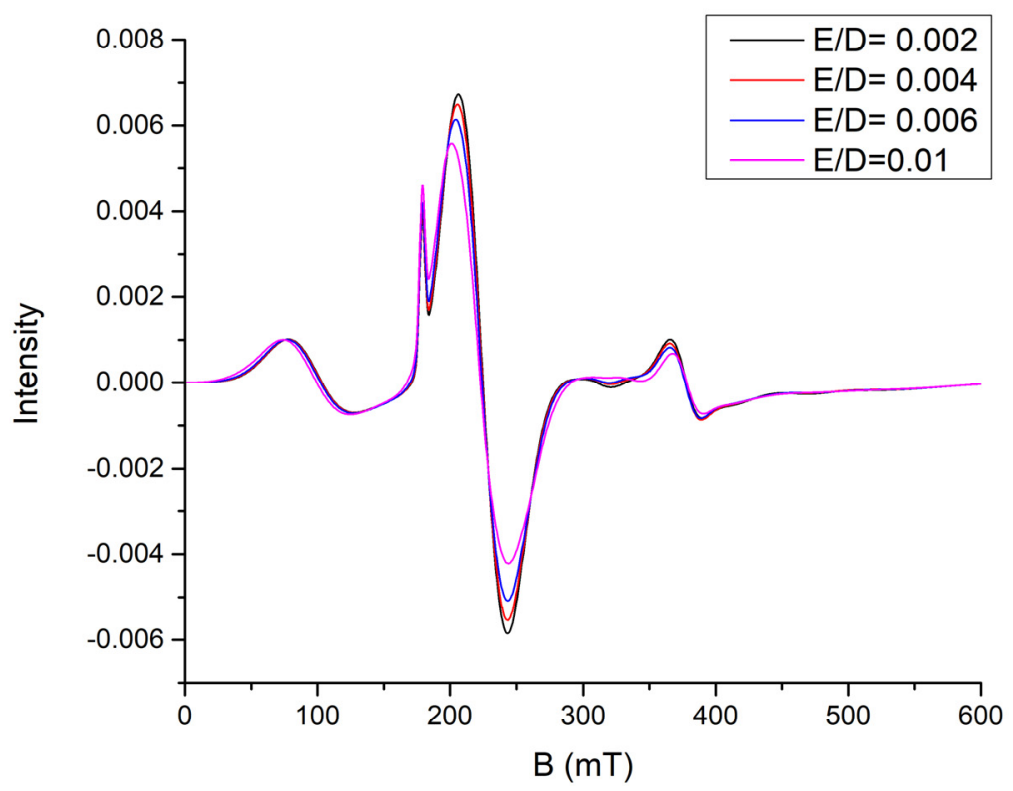

(B)

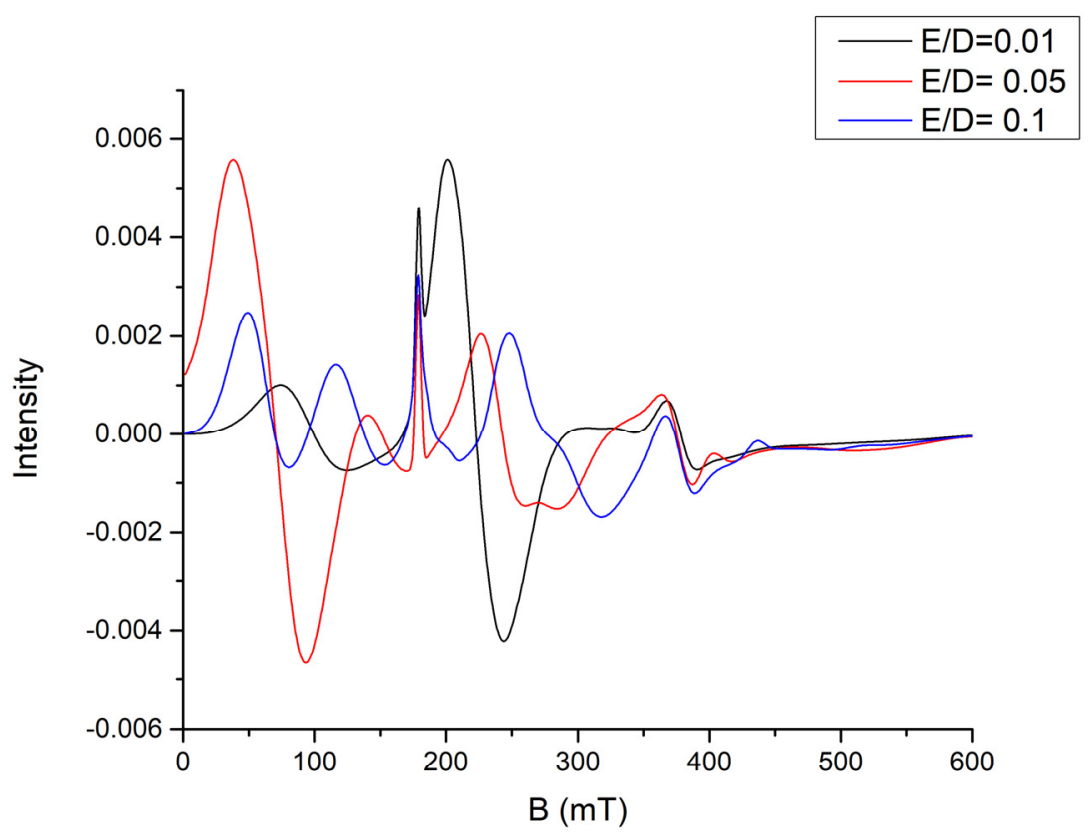

Figure S6. EPR simulations of the Heme 1-3-4 triad. Variation of the $E / D$ ratio of Heme $1(S=5 / 2)$ between 0.002-0.01 (A) and 0.01-0.1 (B). All simulations were conducted with: $D=2.5 \mathrm{~cm}^{-1}, J_{13}=0.334$ $\mathrm{cm}^{-1}$, and $J_{34}=0.001 \mathrm{~cm}^{-1}$. For the $\mathrm{g}$ values used in the simulations see Table 2 . As evident from panels $A$ and $\mathrm{B}$, the simulations do not depend much on the $E / D$ ratio, as long as $E / D<0.01$. 


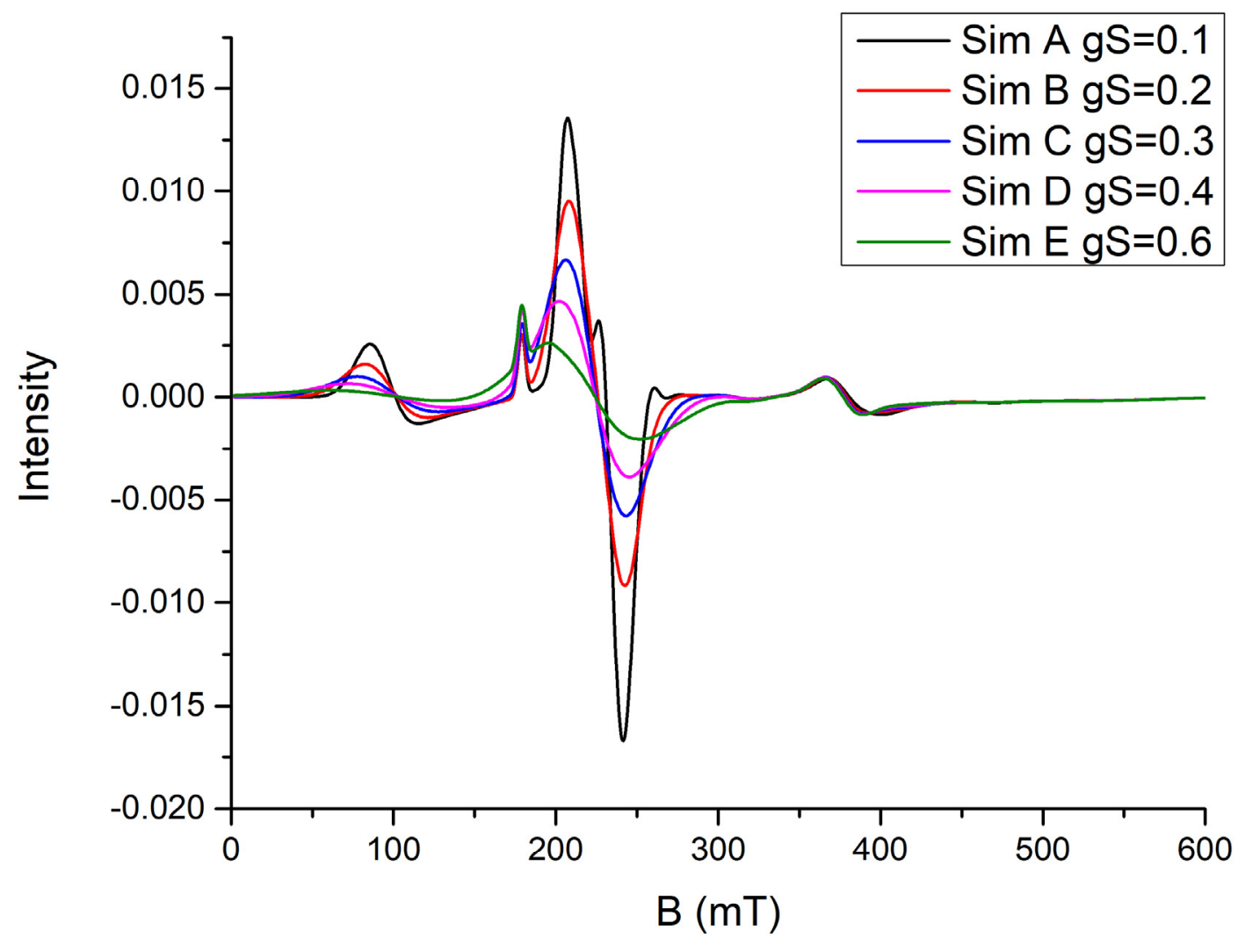

Figure S7. EPR simulations of the Heme 1-3-4 triad. Variation of the Heme $1 \mathrm{~g}$-strain. All simulations were conducted with: $D=2.5 \mathrm{~cm}^{-1}, E=0.005 \mathrm{~cm}^{-1}, E / D=0.002, J_{13}=0.334 \mathrm{~cm}^{-1}$ and $J_{34}=0.001 \mathrm{~cm}^{-1}$. For the $\mathrm{g}$ values used in the simulations see Table 2 . 


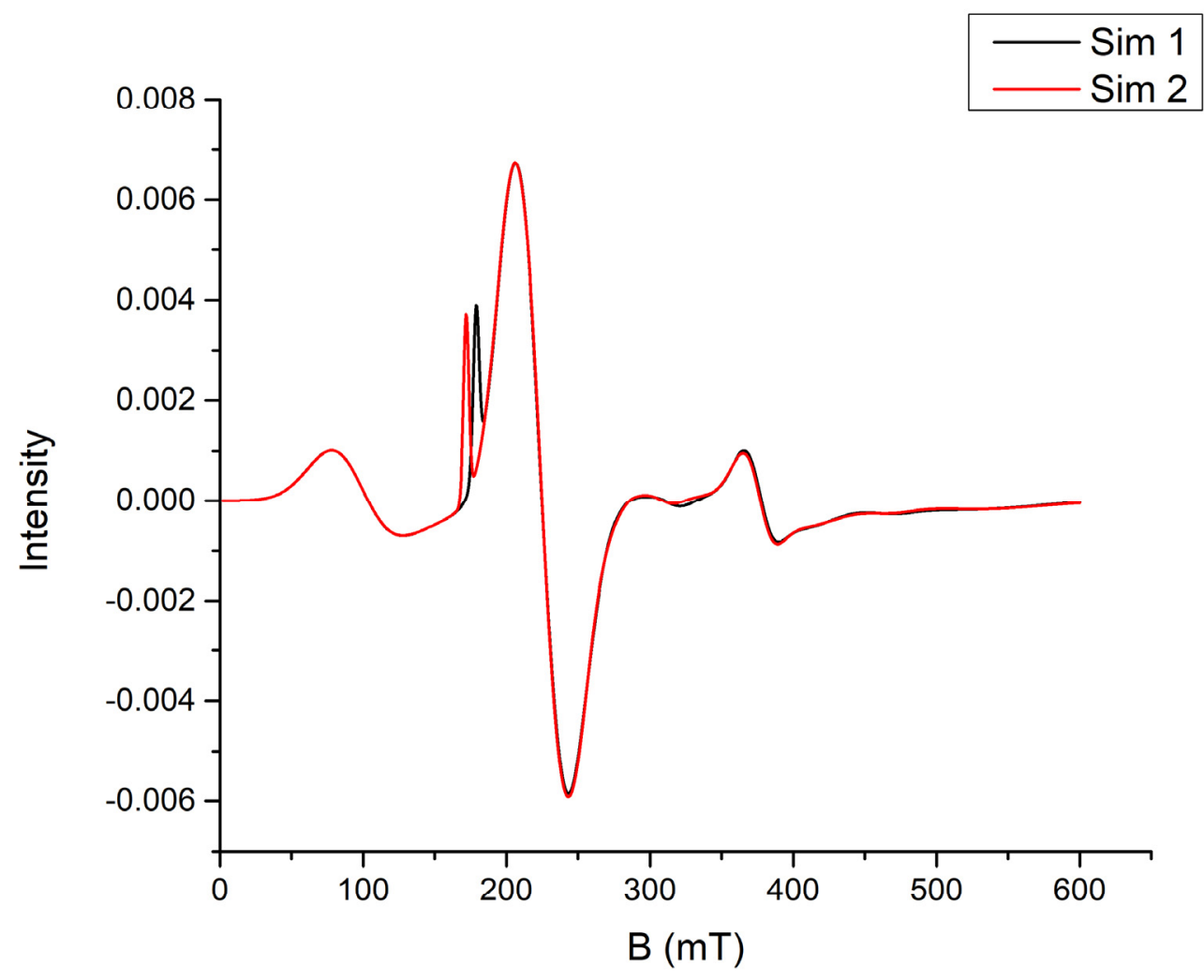

Figure S8. EPR simulations of the Heme 1-3-4 triad. Variation of the $g_{\max (z)}$ value of Heme 4 with $g=3.6$ (Sim1, black) and $\mathrm{g}=3.85$ (Sim2, red). All simulations were conducted with: $D=2.5 \mathrm{~cm}^{-1}, E=0.005 \mathrm{~cm}^{-1}$, $E / D=0.002, J_{13}=0.334 \mathrm{~cm}^{-1}$ and $J_{34}=0.001 \mathrm{~cm}^{-1}$. For the $g$ values used in the simulations see Table 2 . 


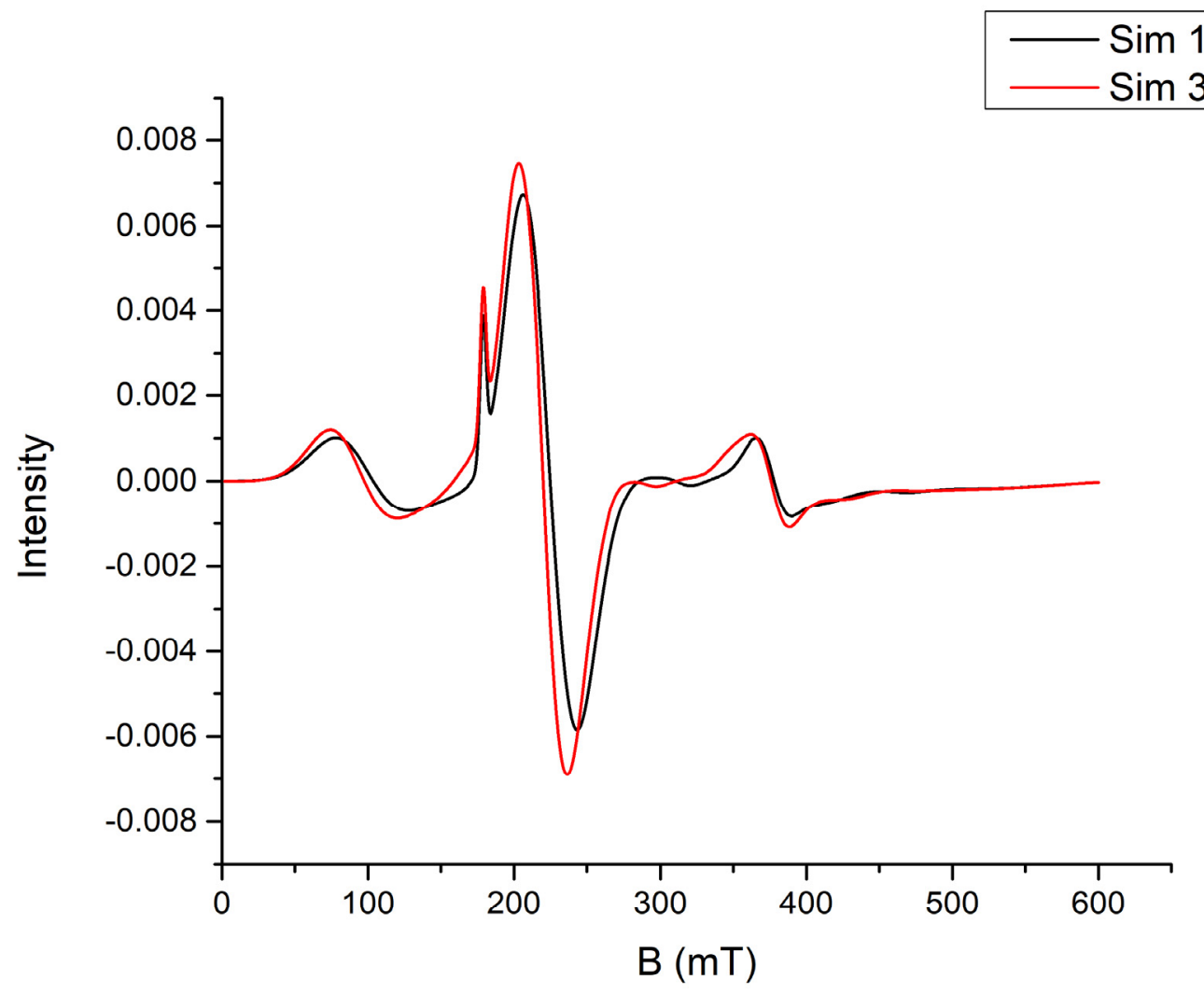

Figure S9. EPR simulations of the Heme 1-3-4 triad. Variation of the Heme 3 parameters with $\mathrm{g}_{\mathrm{z}}=3.22$, $\mathrm{g}_{\mathrm{y}}=2.2$, and $\mathrm{g}_{\mathrm{x}}=1.41$ (Sim1, black) and $\mathrm{g}_{z}=3.42, \mathrm{~g}_{\mathrm{y}}=2.4$, and $\mathrm{g}_{\mathrm{x}}=1.61$ (Sim3, red). Both simulations were conducted with: $D=2.5 \mathrm{~cm}^{-1}, E=0.005 \mathrm{~cm}^{-1}, E / D=0.002, J_{13}=0.334 \mathrm{~cm}^{-1}$ and $J_{34}=0.001 \mathrm{~cm}^{-1}$. The simulated spectrum is not very sensitive to the $\mathrm{g}$ values of Heme 3. However, the EPR parameters of Heme 3 can be determined from the EPR titration (see Figure S12). 


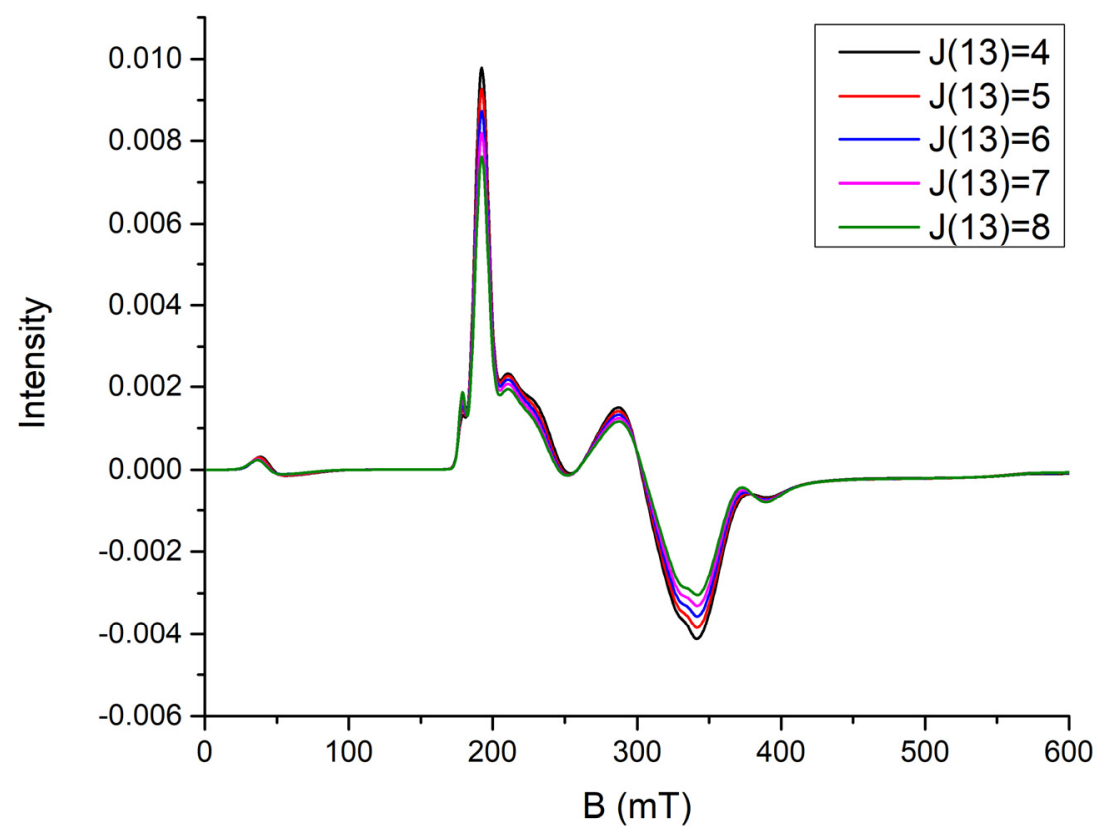

Figure S10. EPR simulations of the Heme 1-3-4 triad where Heme 1 is low-spin $(S=1 / 2)$. Variation of the $J_{13}$ value (coupling of Hemes 1 and 3 ) in the $4-8 \mathrm{~cm}^{-1}$ range. All simulations were conducted with $J_{34}=$ $2.0 \mathrm{~cm}^{-1}$. For the $\mathrm{g}$ values used in the simulations see Table 2 .

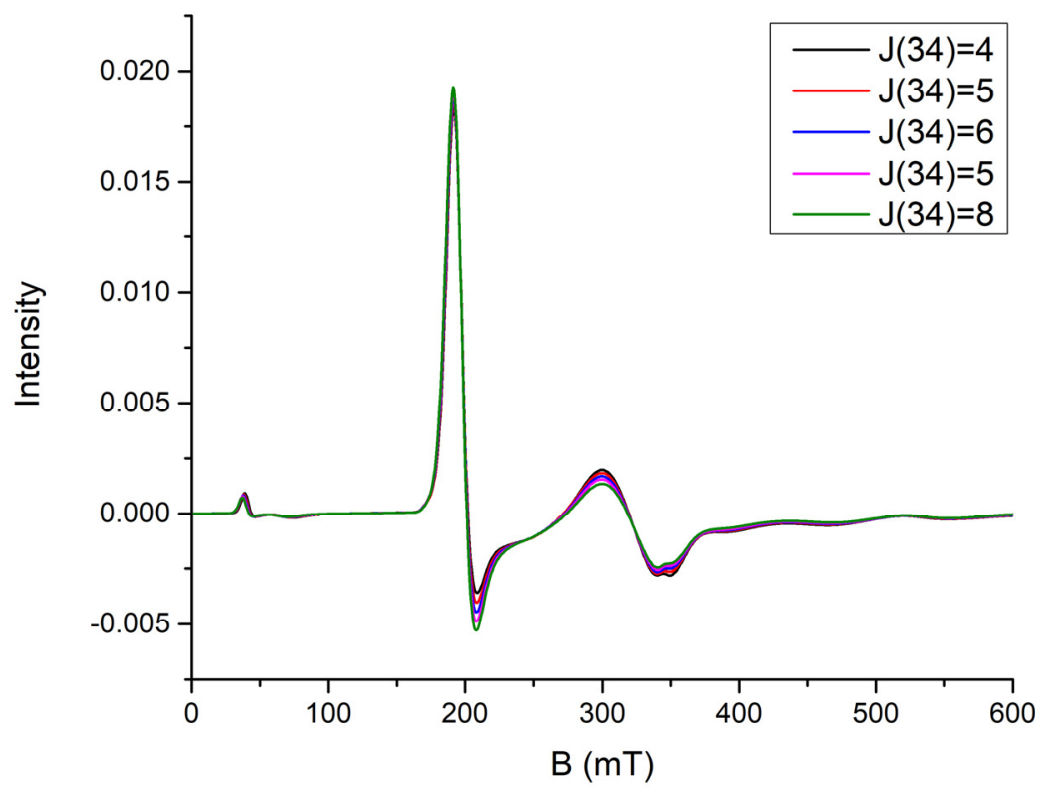

Figure S11. EPR simulations of the Heme 1-3-4 triad where Heme 1 is low-spin ( $S=1 / 2)$. Variation of the $J_{34}$ value (coupling of Hemes 3 and 4 ) in the $4-8 \mathrm{~cm}^{-1}$ range. All simulations were conducted with $J_{13}=$ $0.333 \mathrm{~cm}^{-1}$. For the $\mathrm{g}$ values used in the simulations see Table 2 . 


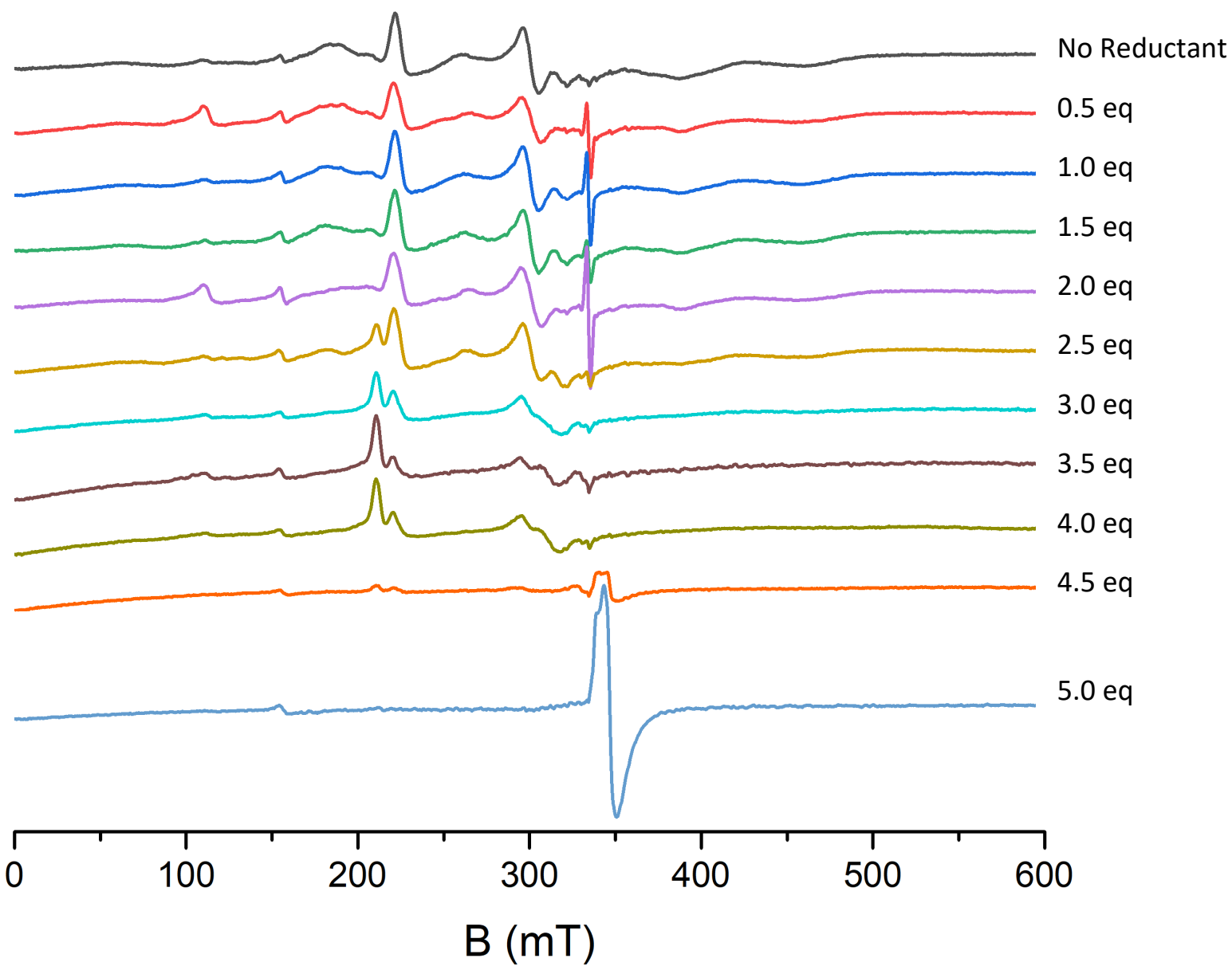

Figure S12. Titration of fully oxidized NrfA in $50 \mathrm{mM}$ HEPES buffer with $150 \mathrm{mM} \mathrm{NaCl}$ at pH 7.0, followed by EPR spectroscopy. Here, the addition of half-equivalents of reductant [Ti(III)(Cit) $\left.{ }_{3}\right]$, up to 5 equivalents, causes characteristic spectral changes that are further analyzed in the text. The large signal observed at $\mathrm{g}=1.9$ upon addition of 5 equiv of reductant corresponds to excess [Ti(III)(Cit) $\left.)_{3}\right](S=1 / 2)$. 
A.
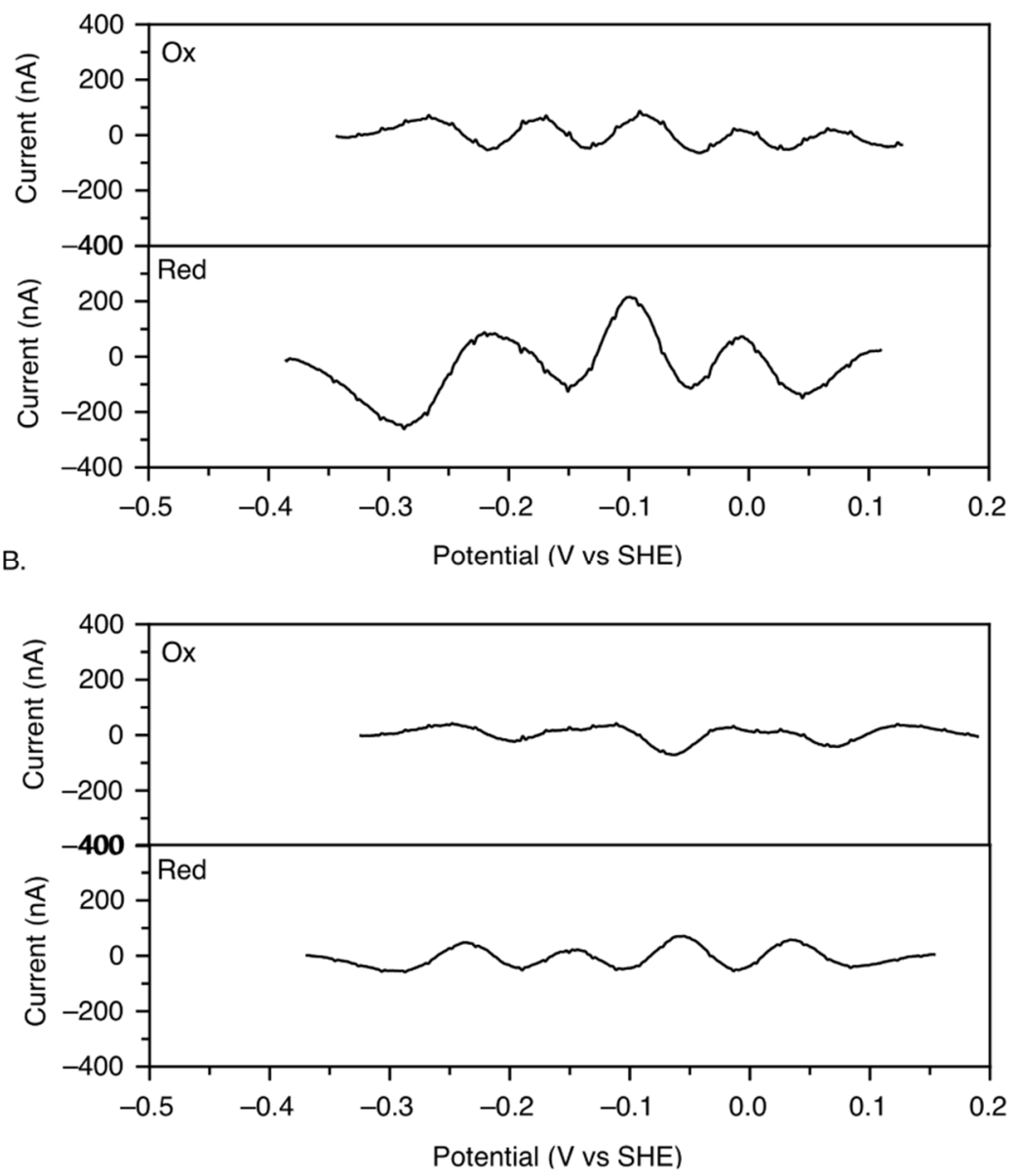

Figure S13. A. Residuals for fits of the oxidative and reductive scans of WT NrfA and B. Residuals for fits of the oxidative and reductive scans of H108M NrfA. Conditions are $\mathrm{pH} 6.0,4{ }^{\circ} \mathrm{C}$ and $10 \mathrm{mV} / \mathrm{sec}$. 


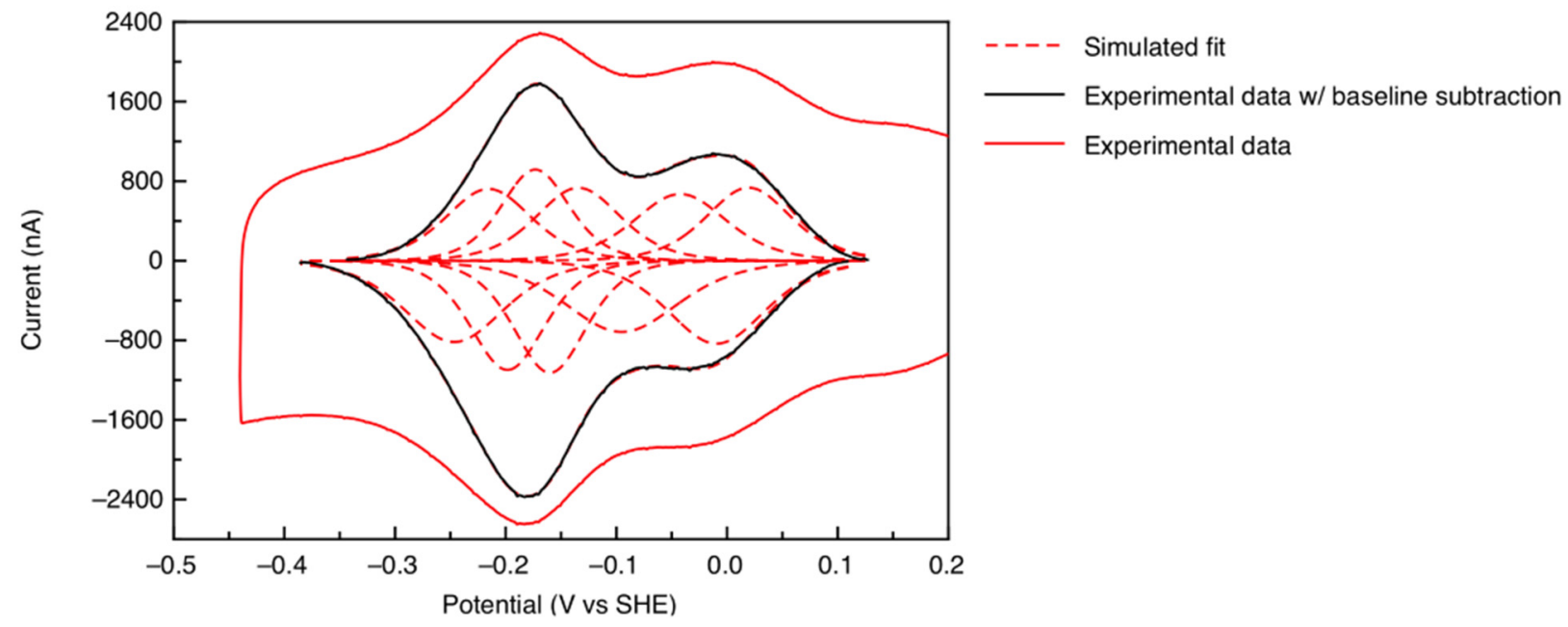

Figure S14. Alternative fits for baseline-subtracted non-turnover G. lovleyi WT NrfA voltammogram ( $\mathrm{pH}$ $6.0,4^{\circ} \mathrm{C}, 10 \mathrm{mV} / \mathrm{sec}$ ) with $\mathrm{n}$ value (electrons transferred per electrochemical event) not fixed at $\mathrm{n}=1$, and instead calculated as a best fit.

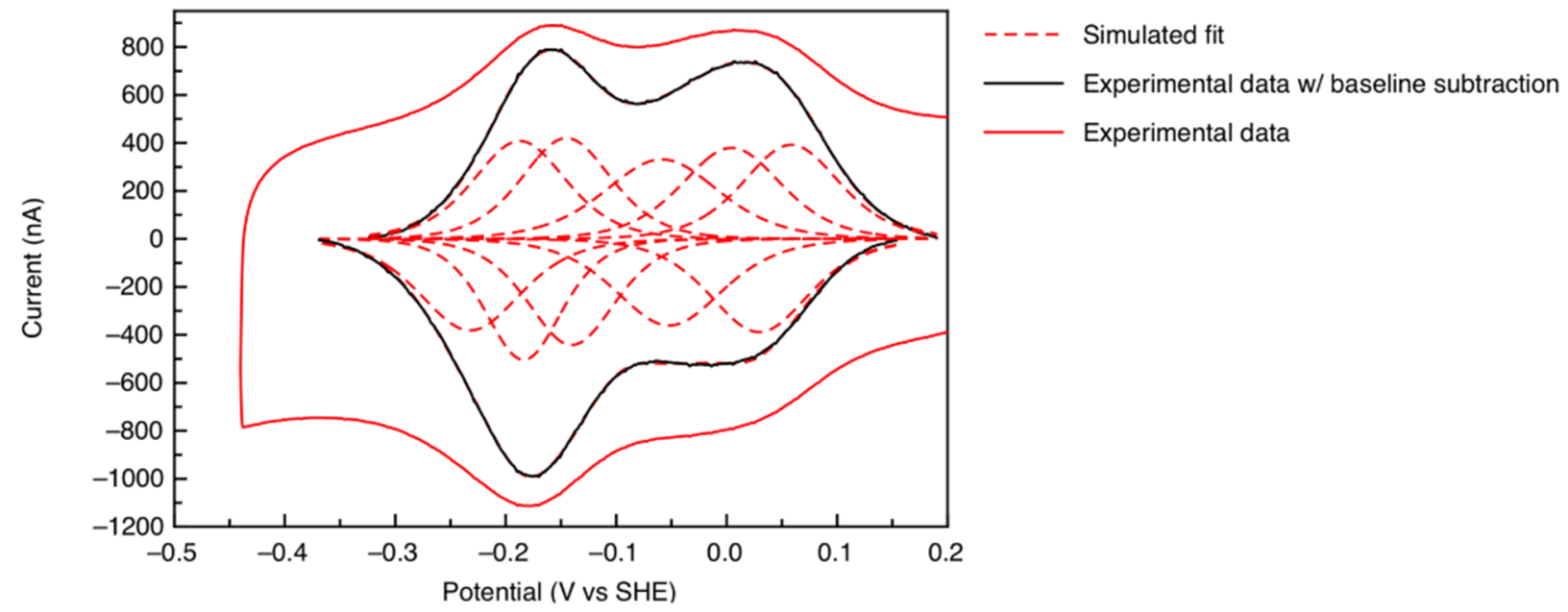

Figure S15. Alternative fits for baseline-subtracted non-turnover G. lovleyi H108M NrfA voltammogram $\left(\mathrm{pH} 6.0,4^{\circ} \mathrm{C}, 10 \mathrm{mV} / \mathrm{sec}\right)$ with $\mathrm{n}$ value (electrons transferred per electrochemical event) not fixed at $\mathrm{n}=$ 1 , and instead calculated as a best fit. 

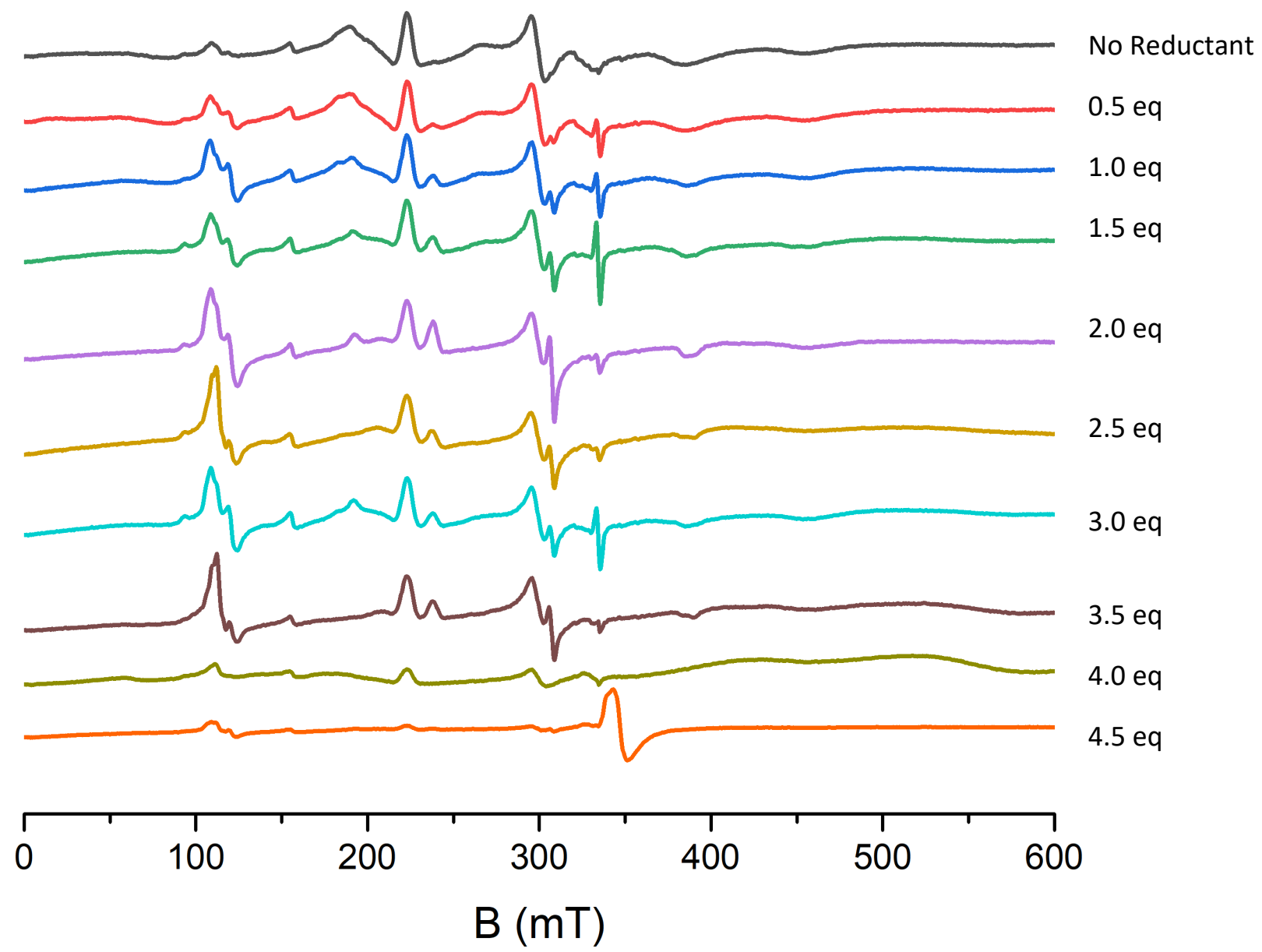

Figure S16. Titration of fully oxidized H108M NrfA in $50 \mathrm{mM} \mathrm{HEPES} \mathrm{buffer} \mathrm{with} 150 \mathrm{mM} \mathrm{NaCl}$ at pH 7.0, followed by EPR spectroscopy. Here, the addition of half-equivalents of reductant [Ti(III)(Cit) 3 ], up to 4.5 equivalents, causes characteristic spectral changes that are further analyzed in the text. The large signal observed at $g=1.9$ upon addition of 4.5 equiv of reductant corresponds to excess [Ti(III)(Cit) 3$](S=1 / 2)$. 


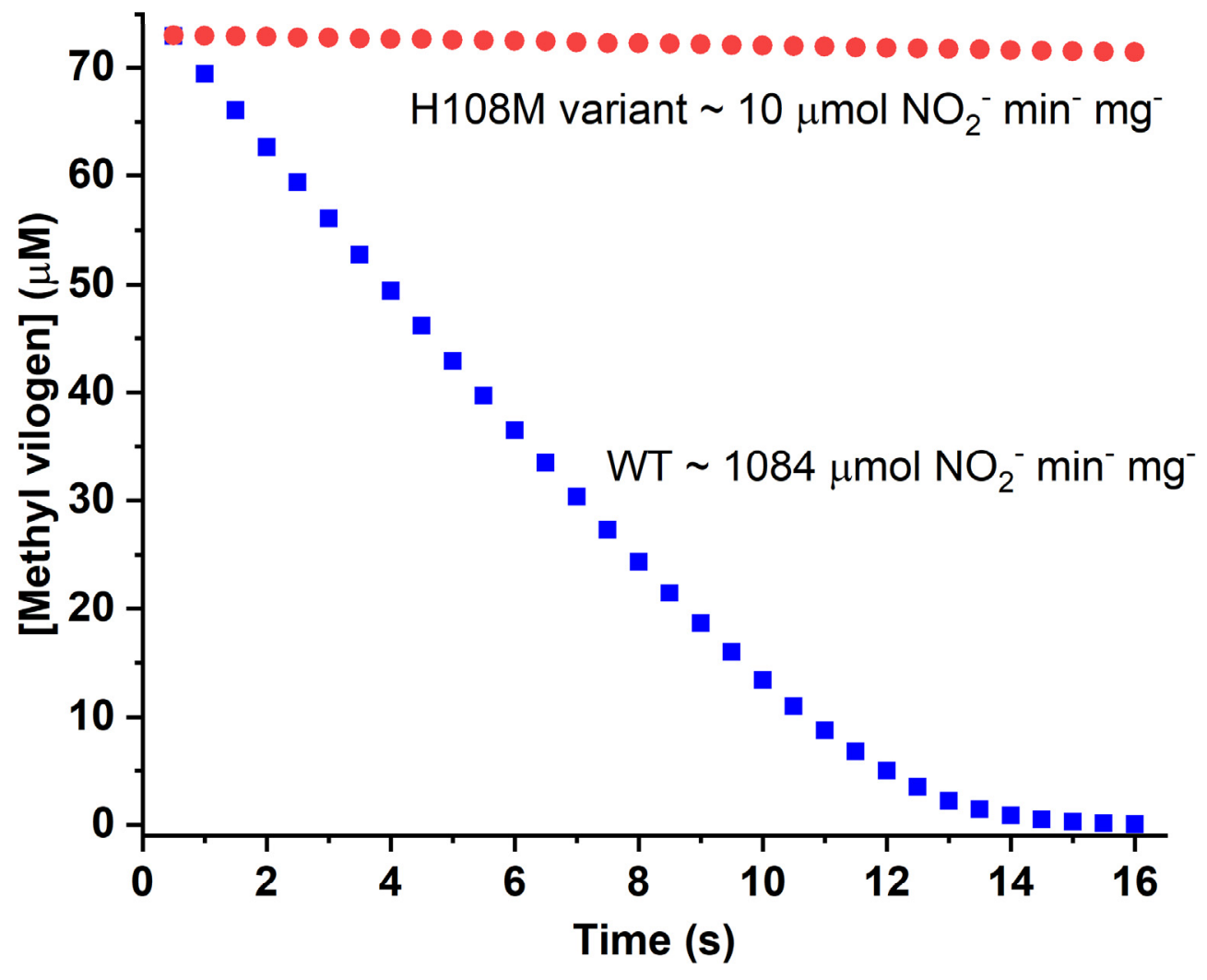

Figure S17. Nitrite reduction activity of the G. lovleyi NrfA H108M variant in comparison to the WT enzyme. 

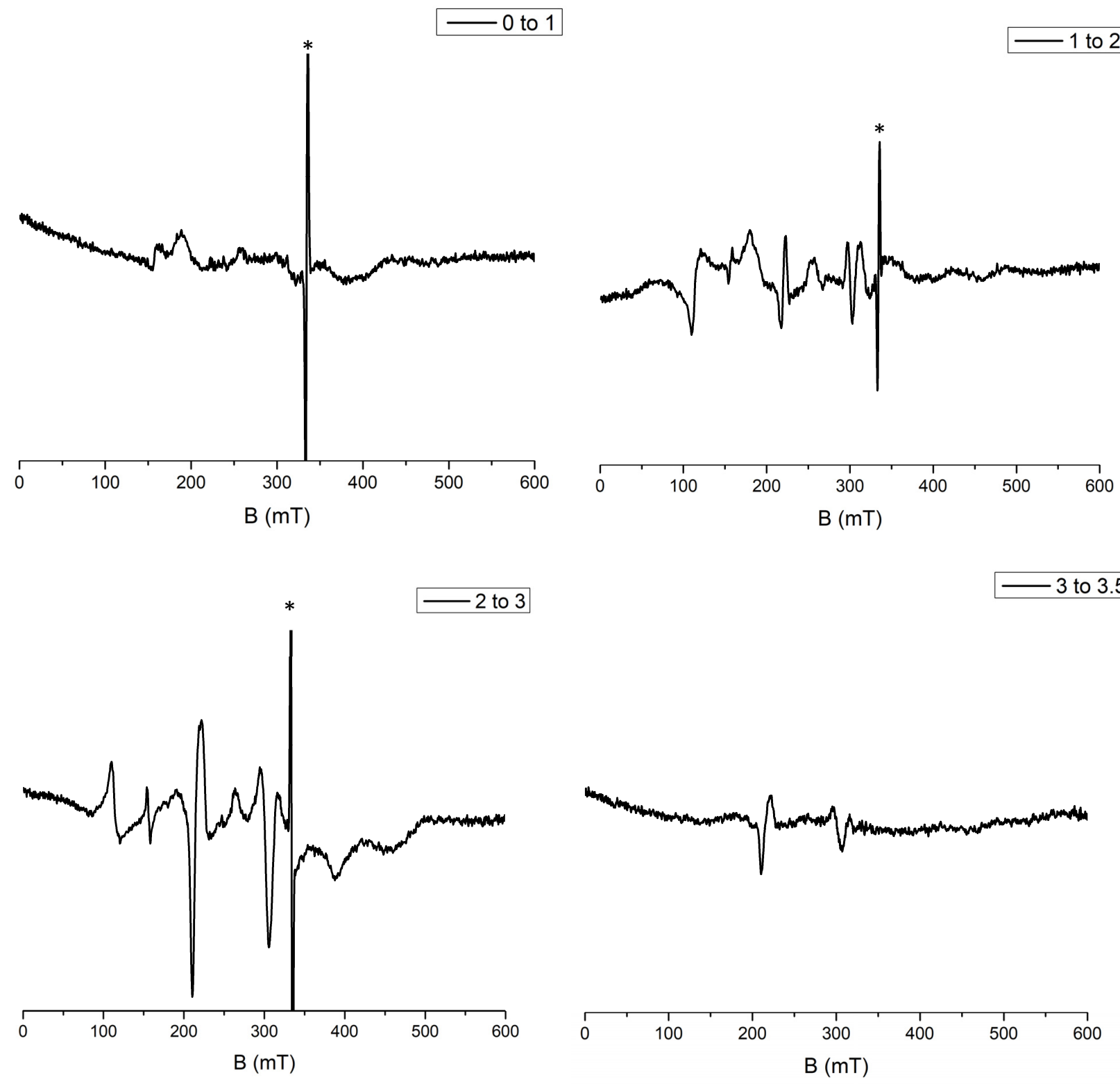

Figure S18. Titration of fully oxidized WT NrfA in $50 \mathrm{mM}$ HEPES buffer with $150 \mathrm{mM} \mathrm{NaCl}$ at pH 7.0, followed by EPR spectroscopy. Here, we show the respective difference spectra from Figure 6 after the addition of halfequivalents of reductant $\left[\mathrm{Ti}(\mathrm{III})(\mathrm{Cit})_{3}\right]$, up to 5 equivalents. This causes characteristic spectral changes that are further analyzed in the text. Spectra shown in the panels correspond to the subtraction of spectra with different equiv of [Ti(III)(Cit) 3 ] added, as indicated: (A) 0 equiv - 1 equiv; (B) 1 equiv - 2 equiv, (C) 2 equiv - 3 equiv, and (D) 3 equiv -3.5 equiv. ${ }^{*}$ The $g=2$ peak is due to a residual radical from the reductant and not the protein. 
(A)
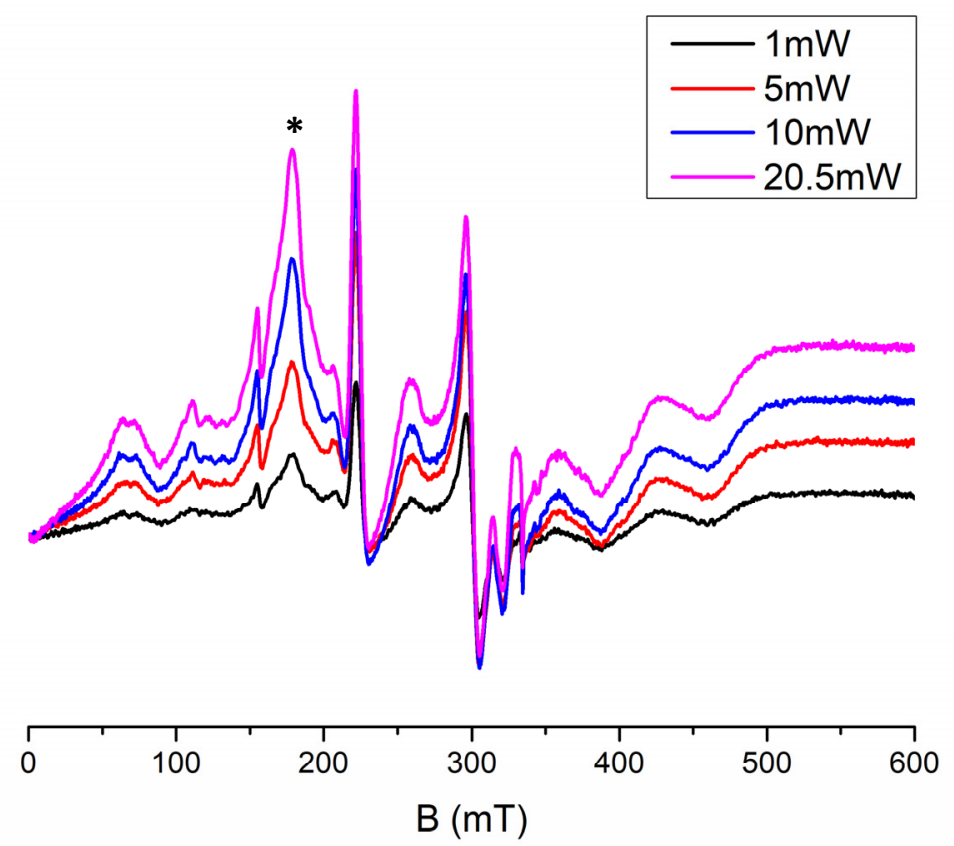

(B)
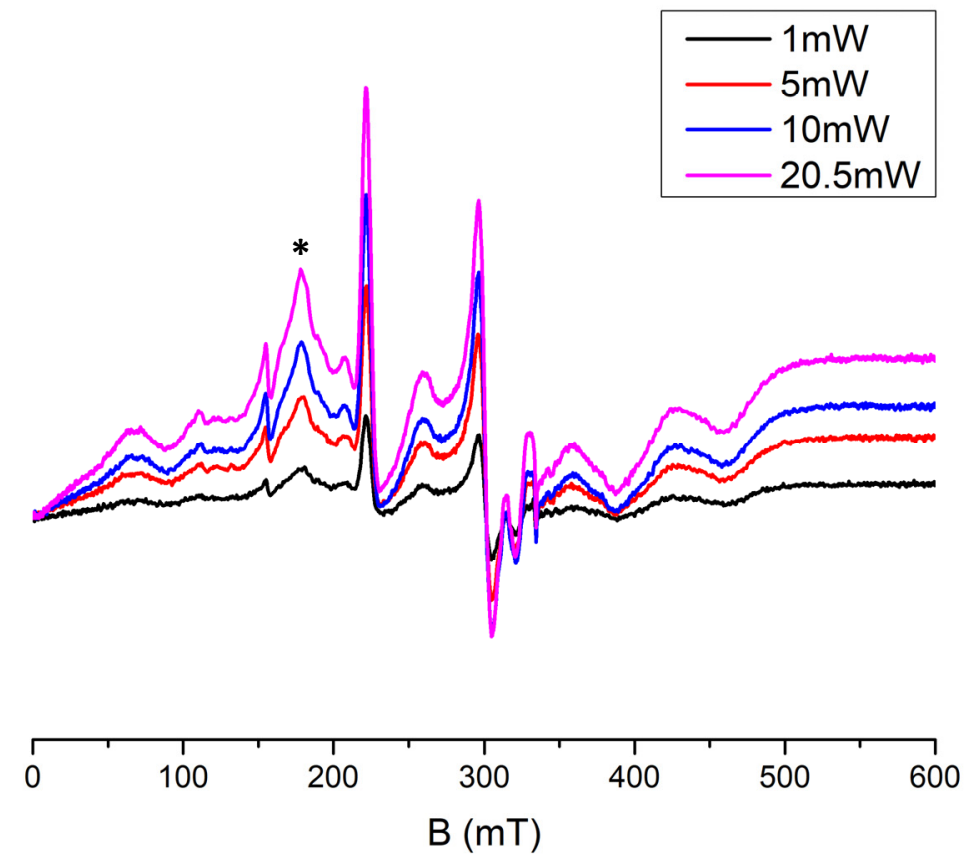

Figure S19. Power dependence of fully oxidized WT NrfA in $50 \mathrm{mM}$ HEPES buffer with $150 \mathrm{mM} \mathrm{NaCl}$ at pH 7.0 EPR signals recorded at different microwave power at (A) $10 \mathrm{~K}$ and (B) 15K. The signals marked with an asterisk (*) correspond to impurities. 
(A)

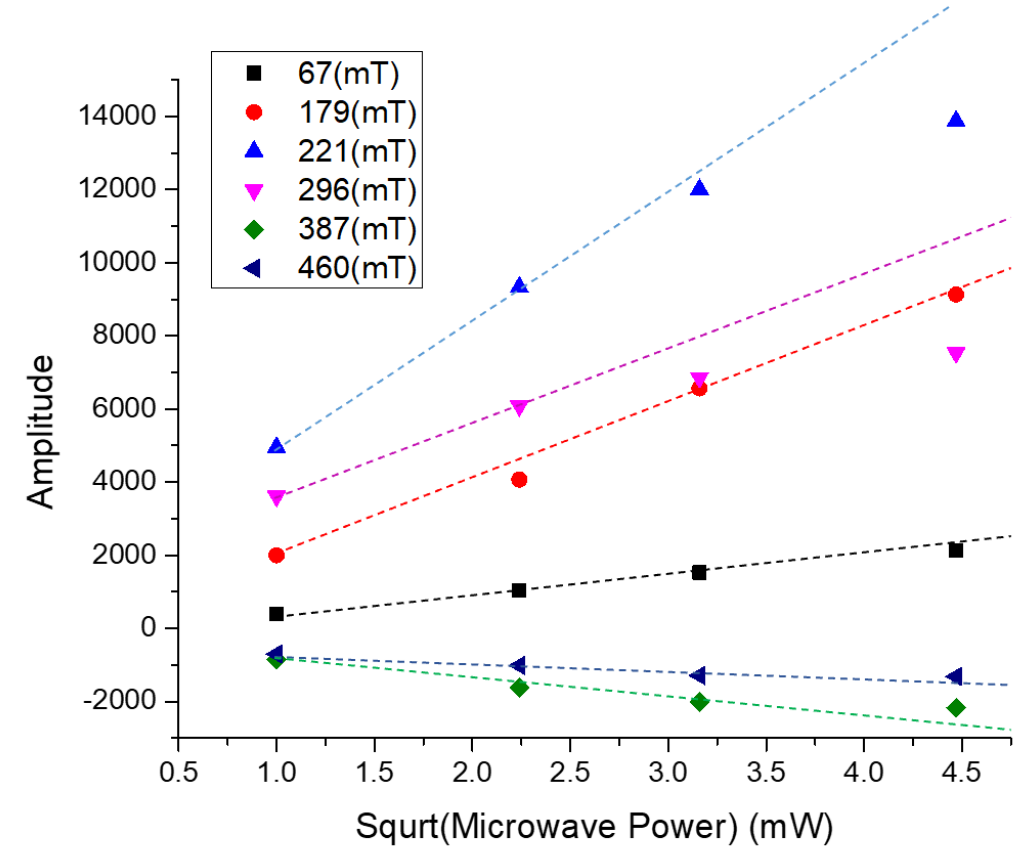

(B)

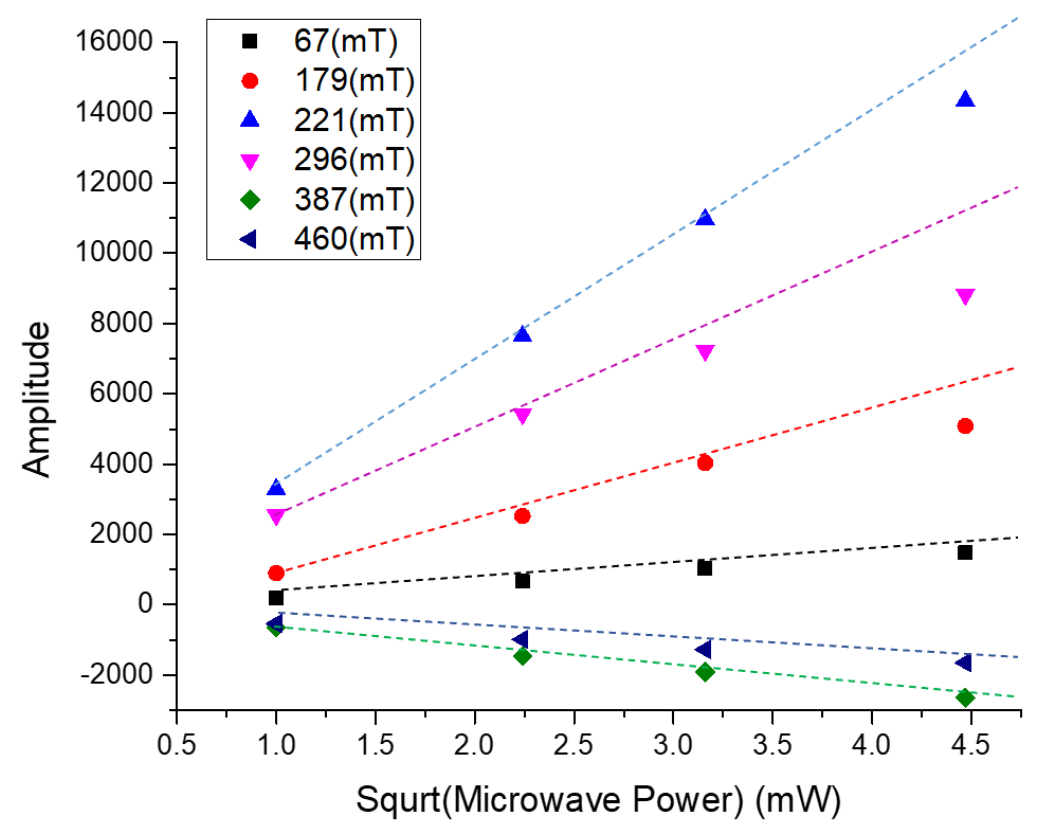

Figure S20. Power dependence plots of fully oxidized WT NrfA in $50 \mathrm{mM} \mathrm{HEPES} \mathrm{buffer} \mathrm{with} 150 \mathrm{mM} \mathrm{NaCl}$ at $\mathrm{pH} 7.0$ at (A) 10K and (B) 15K (generated from Figure S19 data). 


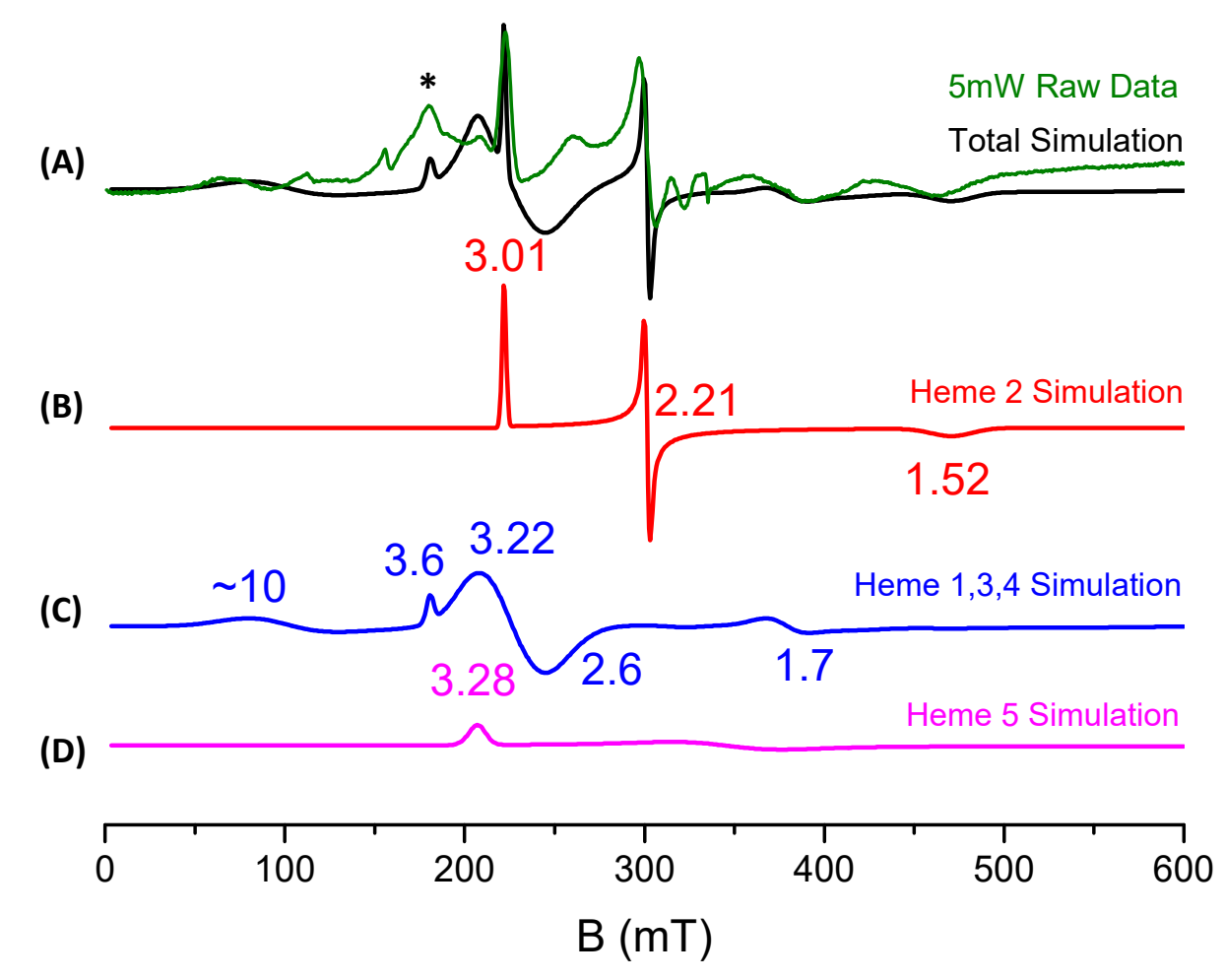

Figure S21. A. Overlay of the EPR spectrum of as-isolated, fully oxidized G. lovleyi NrfA (green line; at microwave power of $5 \mathrm{~mW}$ ) and the total simulation (= sum of the spectra shown in panels B-D, black line). The three simulated components consist of [Heme 2] (red, panel B), [Heme 1 ( $S=5 / 2$ )-Heme 3-Heme 4] (blue, panel C), and [Heme 5] (magenta, panel D). The signal marked with an asterisk $(*)$ contains contributions from impurities as well. 


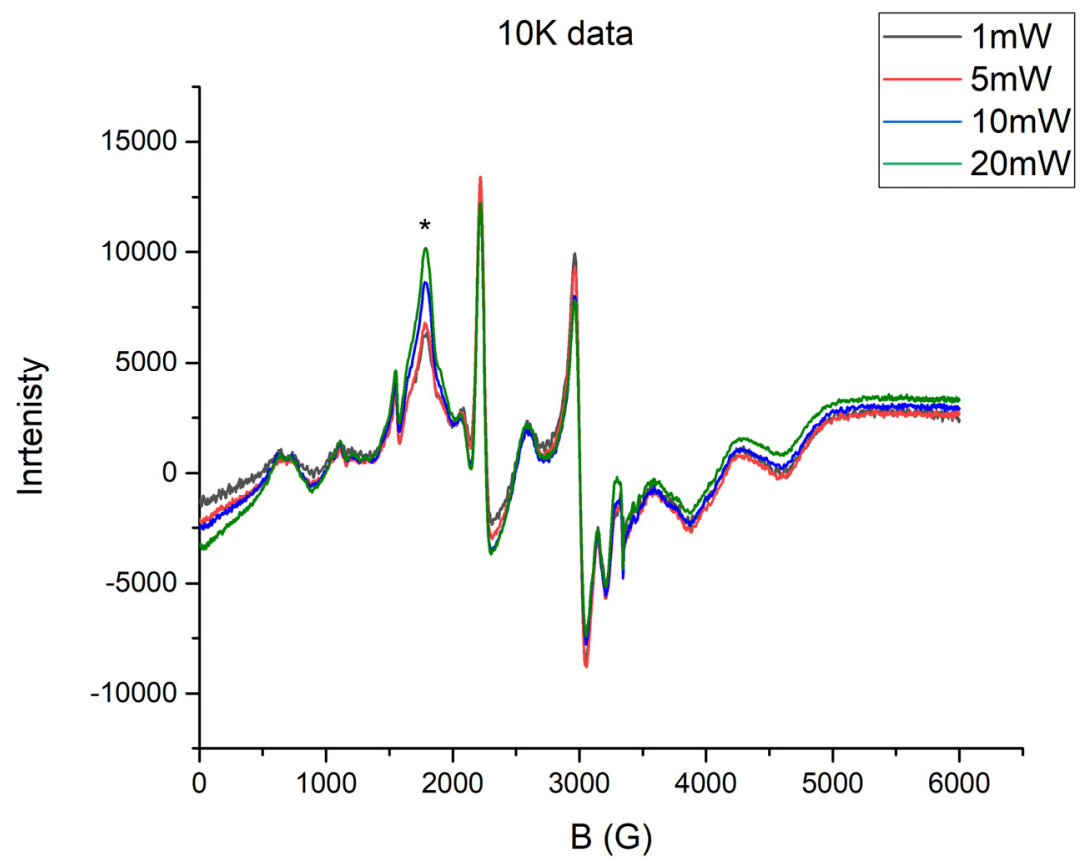

Figure S22. Normalized power-dependent EPR spectra of fully oxidized WT NrfA in 50mM HEPES buffer with $150 \mathrm{mM} \mathrm{NaCl}$ at pH 7.0, measured at $10 \mathrm{~K}$ (data from Figure S19, top). The signal marked with an asterisk $(*)$ corresponds to an impurity (evident from a comparison to the EPR data of highly-purified NrfA in Figure 3 in the manuscript. Here, the spectral intensity in the $g=3.3-3.8$ region is much lower).

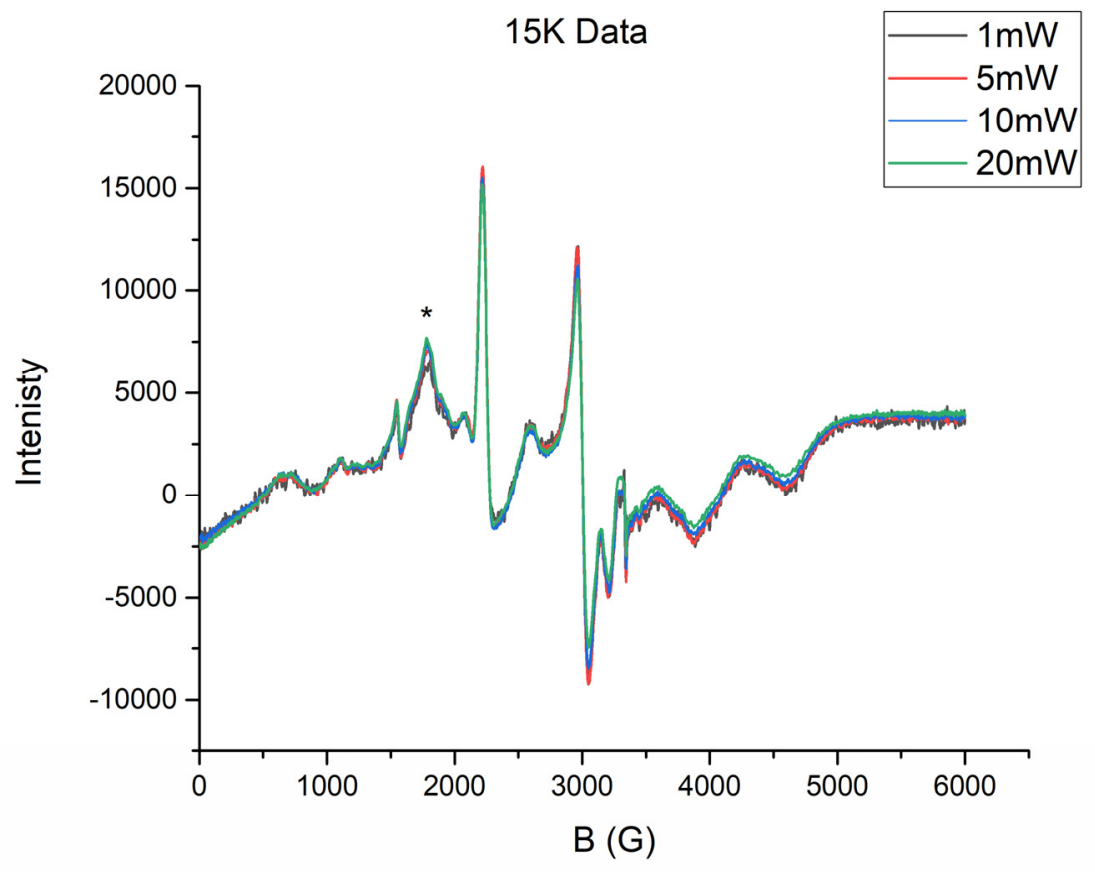

Figure S23. Normalized power-dependent EPR spectra of fully oxidized WT NrfA in 50mM HEPES buffer with $150 \mathrm{mM} \mathrm{NaCl}$ at pH 7.0, measured at $15 \mathrm{~K}$ (data from Figure S19, bottom). The signal marked with an asterisk $\left({ }^{*}\right)$ corresponds to an impurity. 
Table S1. Tabulation of calculated $\mathrm{n}$ values (electrons transferred) for each oxidative and reductive wave for WT NrfA and H108M NrfA.

\begin{tabular}{|l|l|l|l|l|l|}
\hline & $\begin{array}{l}\text { Most negative } \\
\text { peak (peak 1) }\end{array}$ & Peak 2 & Peak 3 & Peak 4 & Peak 5 \\
\hline $\begin{array}{l}\text { Oxidative scan } \\
\text { (wtNrfA) }\end{array}$ & $0.91 \pm 0.007^{\text {a }}$ & $1.16 \pm 0.01$ & $0.93 \pm 0.01$ & $0.85 \pm 0.006$ & $0.93 \pm 0.004$ \\
\hline $\begin{array}{l}\text { Reductive scan } \\
\text { (wtNrfA) }\end{array}$ & $0.79 \pm 0.007$ & $1.09 \pm 0.01$ & $1.06 \pm 0.01$ & $0.69 \pm 0.008$ & $0.81 \pm 0.004$ \\
\hline $\begin{array}{l}\text { Oxidative scan } \\
\text { (H108M NrfA) }\end{array}$ & $0.79 \pm 0.007$ & $0.85 \pm 0.003$ & $0.87 \pm 0.006$ & $0.81 \pm 0.003$ & $0.69 \pm 0.007$ \\
\hline $\begin{array}{l}\text { Reductive scan } \\
\text { (H108M NrfA) }\end{array}$ & $0.91 \pm 0.009$ & $0.78 \pm 0.005$ & $1.03 \pm 0.01$ & $0.74 \pm 0.005$ & $0.80 \pm 0.003$ \\
\hline
\end{tabular}

a. Errors shown are calculated statistical errors in the fitting algorithm used by the ODRPACK module in qSOAS

Table S2. Tabulated midpoint potential values, in $\mathrm{mV}$ vs. SHE, comparing fits with fixed $\mathrm{n}=1$ and fits with free $n$ values, for WT NrfA and H108M NrfA (data collected at $\mathrm{pH} 6.0,4^{\circ} \mathrm{C}$, and $10 \mathrm{mV} / \mathrm{sec}$ )

\begin{tabular}{|l|l|l|l|l|l|}
\hline & $\begin{array}{l}\text { Most negative } \\
\text { peak (peak 1) }\end{array}$ & Peak 2 & Peak 3 & Peak 4 & Peak 5 \\
\hline wtNrfA & -226 & -180 & -152 & -66 & +10 \\
\hline wtNrfA (free n) & -231 & -186 & -147 & -69 & +5 \\
\hline H108M NrfA & -210 & -160 & -103 & -24 & +48 \\
\hline $\begin{array}{l}\text { H108M NrfA } \\
\text { (free n) }\end{array}$ & -210 & -164 & -99 & -24 & +44 \\
\hline
\end{tabular}

